\title{
Robustness Study on the Model-Free Control and the Control with Restricted Model of a High Performance Electro-Hydraulic System
}

\author{
Yaozhong Xu , Eric Bideaux , and Daniel Thomasset \\ Laboratory AMPERE - UMR CNRS 5005, INSA Lyon, University of Lyon, Villeurbanne, France \\ E-mail: yaozhong.xu@insa-lyon.fr, eric.bideaux@insa-lyon.fr, daniel.thomasset@insa-lyon.fr
}

\begin{abstract}
In industry, some unexpected or unpredicted effects make difficult to apply certain control techniques which show good performances in laboratory. In this paper, we introduce two new control methods, called the model-free control and the control with restricted model [1-3], in the case of position tracking of an electro-hydraulic test rig. For an industrial point of view, these methods present many advantages as they are closed to the classic PID control and do not required an intensive modelling work. However, an accurate differentiation of the output has to be done on-line at high sampling frequency in order to estimate accurately the evolution of the state function. Experiments are conducted to estimate the sensibility of these control strategies to the system parameter perturbations. The results illustrate that the proposed controls have a good robustness performance, and that the tracking performance is sensitive to the velocity estimation precision.
\end{abstract}

Keywords: Model-Free Control, Control with Restricted Model, Robustness Analysis, Nonlinear System, Eletro-Hydraulic System

\section{Introduction}

Hydraulic technology has been developed in industry for long ago. Despite the large use of hydraulic system, it is still difficult to achieve an accurate position tracking on a large frequency range due to its nonlinearities [4], such as friction force, servovalve dynamic and flow rate characteristics. These nonlinearities make difficult a precise modelling of the hydraulic system. In previous research, some researchers tend to solve this problem by linearizing the nonlinear state equations to degrade it into a high-order linear model [5]. However, this kind of model is not accurate in a large operating range and is valid only on the neighbourhood of an operating point. Some nonlinear control strategies, such as the technique of backstepping $[6,7]$, and sliding mode [8,9], have been developed in order to realize the precise control of such a nonlinear system. Nevertheless, they are rarely employed in industry because of their requirement of a precise mathematical modelling to achieve an accurate control and their complexity of implementation and controller gains tuning.

In this paper, we will explore the application of the modelfree control method [1-3] which can realize an accurate position control without considering a precise system model. This method introduces a common ultra-local model which can approximate the unknown system dynamics on a very short time interval, rather than to develop a global system model. Moreover, the system parameters are estimated rapidly by gathering the system state information from sensors, and is automatically updated at every time interval. This means it can work automatically without manual interruption once well configured, and then provides a new approach to deal with a complex nonlinear system. It has the advantage to be easy to implement and the tuning of the control law coefficients is simplified compared to other nonlinear methods. To improve the control performance, it is possible to use a limited model, for example without all dynamics developed and nonlinearities, while the unknown part is estimated by the model-free method. This evolution of the model-free control is called the control with restricted model. Since more information is known about the system, this method could provide a better control performance.

Because of its independence for the system model and ease of implementation, the model-free control is a convenient approach for application in industry where systems are much more complex than models in laboratory due to some unpredictable effects, such as changes of the environment. The model accuracy is possibly degraded due to the model misadjustment, or unavailability of some measurements, or also because of the difficulty of the system parameters tuning. When a control strategy is applied in practice, problems may occur and lead to a degraded even unpredictable result. The robustness of a control strategy is thus an important criterion of the performance evaluation. Of course, one of the main difficulty of the robustness analysis is to define properly the parameters to check and their range of variation.

Following this introduction, section 2 will give a brief introduction to the implementation of the model-free on our test 
rig. Experiments will be conducted for the robustness analysis with different loads and precisions of the estimated velocity in section 3 . Finally, section 4 will give some conclusions and perspectives.

\section{Control strategy design}

The system under study is a high performance electrohydraulic test rig developed specifically for research purposes. The configuration of the test rig is shown in fig. 1. This test rig is equipped with two high bandwidth servovalves (MOOG D765) used as a 3-way component for driving each actuator chamber. They are both installed on an intermediate block [10] equipped with accumulators and solenoid valves, which conduct the modulated flow rates from the servovalves to a low friction actuator. The implementation of the modelfree control in this rig and the experimental validation has been carefully investigated in [11].

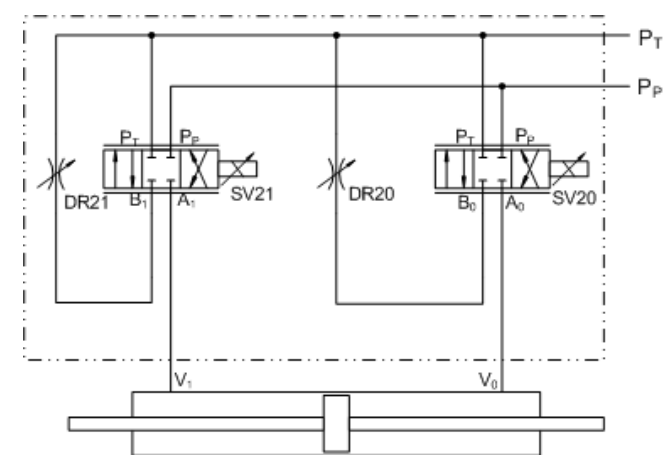

Figure 1: Simplified diagram of electro-hydraulic system

\subsection{Model-Free control}

The electro-hydraulic system is here considered as a SISO (single input single output) system by taking the opposite command condition for the servovalves, namely,

$$
u=u_{1}=-u_{2}
$$

The output of the system is the actuator piston displacement. According to the model-free method, we do not need any information about the system model. In a very short time step (in practice, the sampling period $\Delta T$ ) the system is modelled by

$$
\dot{y}(t)=F(t)+\alpha u(t)
$$

where $F$ represents the unmodelled or time-varying part of the system and will be updated automatically; $\alpha$ is a parameter chosen by the user such that $\alpha u$ and $F$ have the same magnitude.

Then, the value of $F$ is evaluated from $u$ and $\dot{y}$ at any time step as the following:

$$
\widehat{F}_{k}=\widehat{\dot{y}}_{k}-\alpha u_{k-1}
$$

where $\widehat{F}_{k}, \widehat{\dot{y}}_{k}$ are the estimated values of $F$ and $\dot{y}$ at time step $k ; u_{k-1}$ is the command at time step $k-1$. Moreover, $\widehat{\dot{y}}_{k}$ is obtained from position differentiation by applying an adaptive high-order sliding modes differentiator [12].
The controller is then developed from equation (2) as follows:

$$
u_{k}=-\frac{\widehat{F}_{k}}{\alpha}+\frac{\dot{y}_{k}^{d}}{\alpha}+K_{P} e_{k}+K_{I} \sum\left(e_{k} \cdot \Delta T\right)
$$

where $u_{k}$ is the command at time step $k ; \dot{y}_{k}^{d}$ is the desired velocity at time step $k ; e_{k}=y_{k}-y_{k}^{d}$ is the tracking error; $K_{P}$, $K_{I}$ are the classic PI tuning gains.

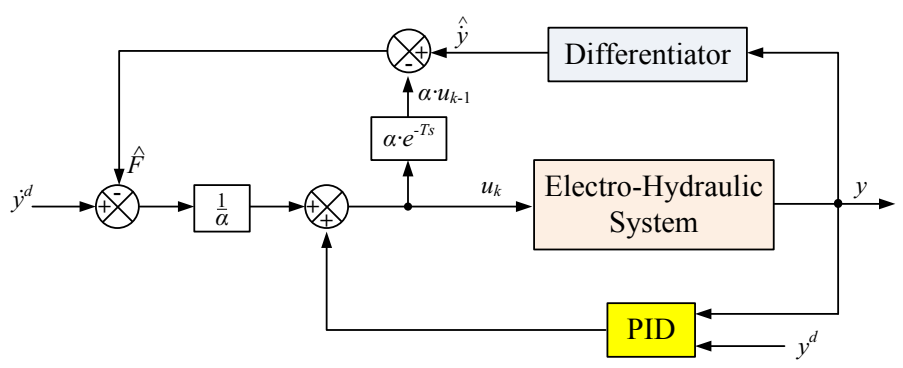

Figure 2: Scheme of the model-free control

The block diagram of the model-free control is presented in figure 2. This controller is called intelligent PI controller or i-PI in $[1,2]$. The last PI part is required to assure the stability of the system and better performances. Compared with a classic PI controller, the i-PI controller contains a part involving the system structure information represented by the estimation of $F$. Therefore, the i-PI can improve the system robustness and tracking performance even for highly nonlinear systems in comparison with the classical PI or without requiring a complex tuning of the parameters as it is the case for nonlinear control laws.

\subsection{Control with restricted model}

A restricted model or an approximate model can be used to improve the pre-calculation of the open-loop command which is applied according to the desired position. In our case, the following assumptions are considered:

1) the internal and external leakages are ignored;

2) the fluid inertia and gravity in each chamber are neglected;

3) the dynamics of the servovalves is neglected;

4) the flow rates are considered proportional to the command.

Since the dynamics of the servovalve has been neglected in the dynamic model for the control design, the servovalve model consists therefore only in a static characteristic described by (6). This formulation is proposed by [13] for simplifying the flow rate calculation. It is related to the supply pressure $P_{P}$, tank pressure $P_{T}$, actuator chamber pressure $P_{1}$ or $P_{2}$, and input command $u_{1}$ or $u_{2}$. In order to get a unified control model, we use an operator sign(.) to couple the inlet and outlet flow in (6). While handling the approximation of the control model, the focus is given on the dynamic of the actuator.

The compressibility of the fluid gives the pressure dynamics in each actuator chamber according to the piston velocity and the flow provided by each servovalve $\left(\phi_{1} u_{1}\right.$ and $\left.\phi_{2} u_{2}\right)$. The 
Newton's law gives the dynamics of the moving load. Dry friction $F_{f s}$ calculated by (7), as well as the viscous friction term and gravity, are taken into account. The dynamic model of the actuator is then as follows:

$$
\left\{\begin{array}{l}
\frac{d y}{d t}=v \\
\frac{d v}{d t}=\frac{A}{M}\left(P_{1}-P_{2}\right)-g-\frac{b}{M} v-\frac{F_{f s}(v)}{M}-\frac{F_{e x t}}{M} \\
\frac{d P_{1}}{d t}=-\frac{\beta}{V_{1}(y)} \cdot A \cdot v+\frac{\beta}{V_{1}(y)} \cdot \phi_{1}\left(P_{1}, P_{P}, P_{T}, \operatorname{sign}\left(u_{1}\right)\right) \cdot u_{1} \\
\frac{d P_{2}}{d t}=\frac{\beta}{V_{2}(y)} \cdot A \cdot v+\frac{\beta}{V_{2}(y)} \cdot \phi_{2}\left(P_{2}, P_{P}, P_{T}, \operatorname{sign}\left(u_{2}\right)\right) \cdot u_{2}
\end{array}\right.
$$

$\phi_{i}=\frac{Q_{n}}{u_{n}} \cdot \sqrt{\frac{\left|P_{P}-P_{i}\right|}{\Delta P_{n}}} \cdot \frac{\operatorname{sign}\left(u_{i}\right)+1}{2}+\frac{Q_{n}}{u_{n}} \cdot \sqrt{\frac{\left|P_{i}-P_{T}\right|}{\Delta P_{n}}} \cdot \frac{\operatorname{sign}\left(u_{i}\right)-1}{2}$

$$
F_{f s}(v)=\left(F_{C}+\left(F_{s d y n}-F_{C}\right) e^{-C|v|}\right) \tanh (v)
$$

For the design of the control with restricted model, a diffeomorphism is firstly required from the initial system coordinates (5) $\left(y, v, P_{1}, P_{2}\right)$ to the new coordinates defined as $\left(y, v, a, P_{1}\right)$. The new system equations are written straightforwardly as

$$
\left\{\begin{aligned}
\frac{d y}{d t}= & v \\
\frac{d v}{d t}= & a \\
\frac{d a}{d t}= & \frac{1}{M}\left(-b \cdot a-\frac{\partial F_{f s}(v)}{\partial v} \cdot a-A^{2} \beta\left(\frac{1}{V_{1}(y)}+\frac{1}{V_{2}(y)}\right) \cdot v\right. \\
& \left.\quad+A \beta\left(\frac{\phi_{1}(\cdot)}{V_{1}(y)} \cdot u_{1}-\frac{\phi_{2}(\cdot)}{V_{2}(y)} \cdot u_{2}\right)\right) \\
\frac{d P_{1}=}{d t}= & -\frac{\beta}{V_{1}(y)} \cdot A \cdot v+\frac{\beta}{V_{1}(y)} \cdot \phi_{1}\left(P_{1}, P_{P}, P_{T}, \operatorname{sign}\left(u_{1}\right)\right) \cdot u_{1}
\end{aligned}\right.
$$

As we consider only a SISO system with the model-free based controls, the pressures are not directly controlled. Instead, the opposite command condition as given by equation (1) is taken into account. Then, this system is degraded to a SISO one with an input command $u$ and a position output $y$ as described in (9).

$$
\left\{\begin{aligned}
\frac{d y}{d t}= & v \\
\frac{d v}{d t}= & a \\
\frac{d a}{d t}= & \frac{1}{M}\left(-b a-\frac{\partial F_{f s}(v)}{\partial v} a-A^{2} \beta\left(\frac{1}{V_{1}(y)}+\frac{1}{V_{2}(y)}\right) v\right. \\
& \left.+A \beta\left(\frac{\phi_{1}(\cdot)}{V_{1}(y)}+\frac{\phi_{2}(\cdot)}{V_{2}(y)}\right) \cdot u\right)
\end{aligned}\right.
$$

By inverting the dynamics model (9), an open-loop nominal command is given by

$$
u^{*}=\frac{M \cdot j^{d}+b \cdot a^{d}+\frac{\partial F_{f s}\left(v^{d}\right)}{\partial v^{d}} \cdot a^{d}+A^{2} \beta\left(\frac{1}{V_{1}\left(y^{d}\right)}+\frac{1}{V_{2}\left(y^{d}\right)} \cdot v^{d}\right)}{A \beta\left(\frac{\phi_{1}(\cdot)}{V_{1}\left(y^{d}\right)}+\frac{\phi_{2}(\cdot)}{V_{1}\left(y^{d}\right)}\right)}
$$

As the system model is incomplete and there exists some unknown effects, the control law can then be defined as

$$
u=u^{*}+\Delta u
$$

where $\Delta u$ represents the compensation command of the unknown phenomena or unmodelled parts of the system and also the feedback controller.
We assume that the compensation command complies with the following relationship (12) with the system output.

$$
\dot{y}(t)=F(t)+\alpha \Delta u(t)
$$

Similarly to what was done with the model-free control, the function $F$ is given by

$$
\widehat{F}_{k}=\widehat{\dot{y}}_{k}-\alpha \Delta u_{k-1}
$$

where $\Delta u_{k-1}=u_{k-1}-u_{k-1}^{*}$

$u_{k-1}^{*}$ is the nominal command at time step $k-1$.

The compensation command at time step $\mathrm{k}$ is then calculated by

$$
\Delta u_{k}=-\frac{\widehat{F}_{k}}{\alpha}+\frac{\dot{y}_{k}^{d}}{\alpha}+K_{P} e_{k}+K_{I} \sum\left(e_{k} \cdot \Delta T\right)
$$

Finally, the controller with restricted model is given in the following equation (16):

$$
u_{k}=u_{k}^{*}-\frac{\widehat{F}_{k}}{\alpha}+\frac{\dot{y}_{k}^{d}}{\alpha}+K_{P} e_{k}+K_{I} \sum\left(e_{k} \cdot \Delta T\right)
$$

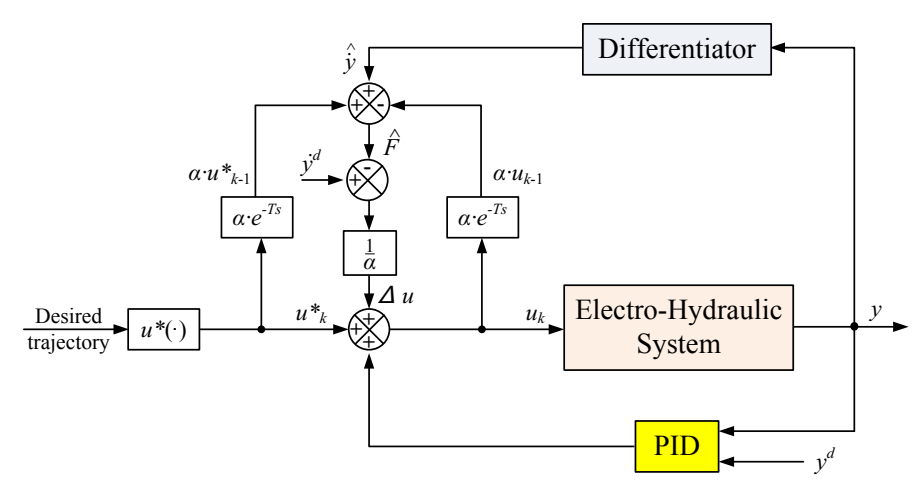

Figure 3: Scheme of the control with restricted model

The block diagram of the model-free control is presented in figure 3. With more information about the system, we will show that the control with restricted model can achieve better control performances than the model-free control, the i-PI being dedicated to the regulation along the tracked trajectory in this case. Some simulation and experimental results are presented in the following sections.

\section{Experimental results}

Experiments are conducted with different loads and different precisions of the estimated velocity in order to study the robustness performance with respect of the mass perturbation of the moving part and the velocity error. The piston position is measured by a LVDT sensor. This position signal is collected into dSPACE acquisition board (DS1104) at a sampling frequency of $1 \mathrm{KHz}$. The velocity information is evaluated by an on-line 2nd-order adaptive differentiators (DAO2) [12]. The desired trajectory is defined by a 7th-order polynomial function which has a smooth velocity, acceleration, and jerk profile.

Some parameters of the test bench are given in table 1 . 
Table 1: Test bench characteristics

\begin{tabular}{|lc|}
\hline \multicolumn{2}{|c|}{ Actuator } \\
Total stroke & $300 \mathrm{~mm}$ \\
Mass of the moving part (rod+piston): $M$ & $5.8 \mathrm{Kg}$ \\
Active section area: $A$ & $9.456 \mathrm{~cm}^{2}$ \\
Dead volume at each end & $14.184 \mathrm{~cm}^{3}$ \\
\hline \multicolumn{2}{|c|}{ Servovalve Moog D765 } \\
Rated flow & $19 \mathrm{~L} / \mathrm{min}$ \\
Rated input signal & $\pm 10 \mathrm{~V}$ \\
Response time & $2 \mathrm{~ms}$ \\
\hline \multicolumn{2}{|c|}{ LVDT sensor installed on the rod of the actuator } \\
Measurement range (MR) & $300 \mathrm{~mm}$ \\
Sensibility & $4.4 \mathrm{mV} / \mathrm{V} / \mathrm{mm}$ \\
Precision & $<0.1 \%$ of the MR \\
\hline
\end{tabular}

\subsection{Experiments with different loads}

In practice, the operating situation is always changing according to the task requirements, e.g. the loads, the environmental conditions corresponding to different tasks. A good control strategy with high performance should show insensitivity to these changes. In this section, we will mainly discuss the influence of the payload change on the control performances. Experiments are conducted with different loads in order to investigate the robustness performance with respect to the mass perturbation. The load will vary from 0 to $20 \mathrm{~kg}$, while the other experimental configurations remain unchanged.

In order to make a comparison with the classic linear control strategy, we present firstly the experimental results obtained by a proportional control with different loads in figure 4 . The experimental results of the proportional control with a load of $20 \mathrm{~kg}$ are not presented in the figure as its behaviour becomes unstable when the load reaches $20 \mathrm{~kg}$. From this figure, we can observe that the position tracking curve in the experiment with a load of $10 \mathrm{~kg}$ presents already oscillations with an important amplitude up to $1 \mathrm{~mm}$, which implied that the control tended to make the controlled whole system unstable.

Since the static error is very low, only the gain $K_{P}$ is really required. The loads are 0,10 , and $20 \mathrm{~kg}$, and a value of 30 $\mathrm{V} / \mathrm{m}$ is used for the tuning gain $K_{P}$. Figures 5 and 6 present the experimental results obtained respectively by the modelfree control and the control with restricted model.

Compared to the results obtained with the proportional control (figure 4) which becomes unstable at the end of the transient phase, the model-free based control strategies are keeping a good behaviour despite the load changes. Especially, it can be noticed that the accuracy of the position tracking is conserved with the control with restricted model when the payload is increased. No notable changes in the results with the different loads can be observed, which implies that the mass change has little influence on the tracking performance of the proposed strategies. This is due to the self-adjustment of the control strategies according to the estimation of the uncertain ultra-local parameter $F$.

Table 2 gives a summary of the position errors obtained with both the model-free control and the control with restricted

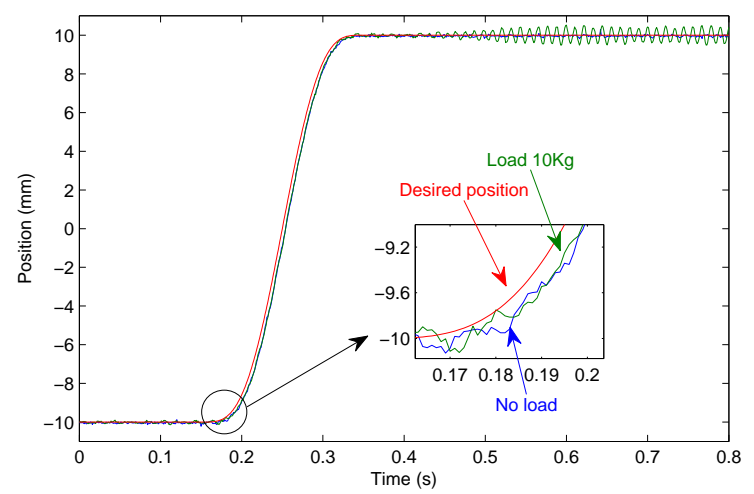

(a) Position tracking

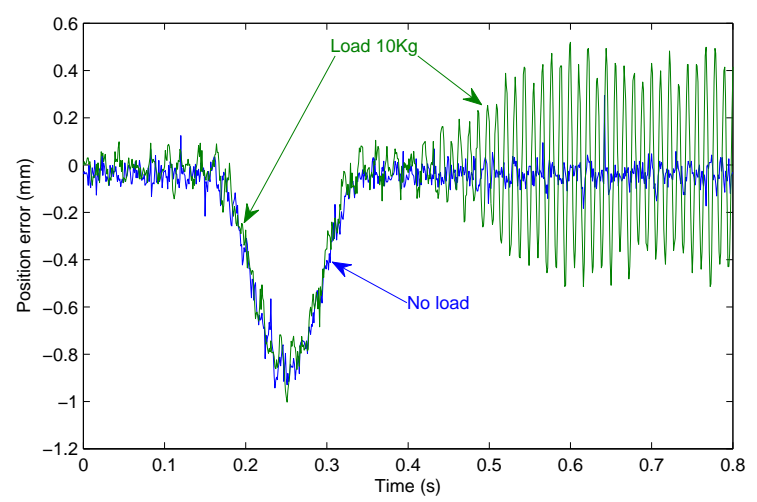

(b) Position error

Figure 4: Robustness experiment results obtained by the proportional control

Table 2: Comparison summary of the position error $(\mu \mathrm{m})$ with different loads in model-free control (MFC), control with restricted model (CRM), and proportional control (Prop.)

\begin{tabular}{|c|r|r|r|r|r|}
\hline Control & \multicolumn{1}{|c|}{$\begin{array}{c}\text { Load } \\
\text { strategy }\end{array}$} & \multicolumn{2}{|c|}{ Transient phase } & \multicolumn{2}{c|}{ Steady state } \\
\cline { 3 - 6 } & Max. & STD $^{a}$ & Mean & STD $^{a}$ \\
\hline & 0 & 2122 & 1344 & 0.06 & 39 \\
MFC & 10 & 2082 & 1370 & -0.2 & 40 \\
& 20 & 2079 & 1372 & -0.02 & 34 \\
\hline \multirow{4}{*}{ CRM } & 0 & 192 & 71 & 0.2 & 41 \\
& 10 & 189 & 77 & -0.4 & 40 \\
& 20 & 184 & 66 & 0.02 & 33 \\
\hline \multirow{2}{*}{ Prop. } & 0 & 943 & 304 & -41 & 35 \\
& 10 & 1003 & 315 & -10 & 52 \\
\hline
\end{tabular}

a) STD: Standard deviation 


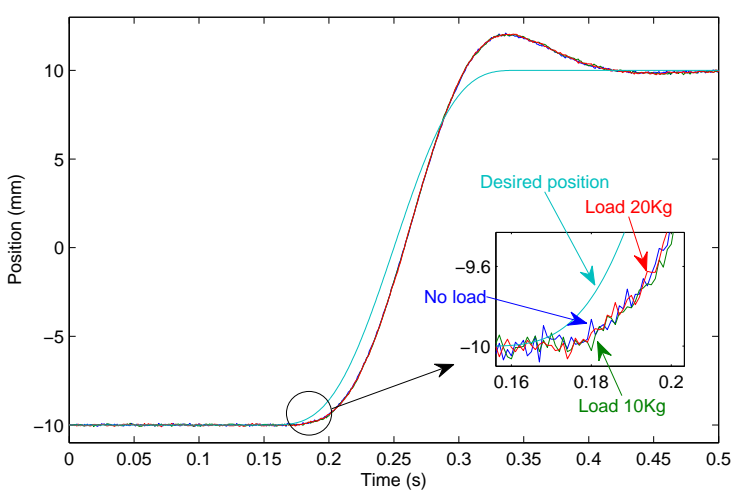

(a) Position tracking

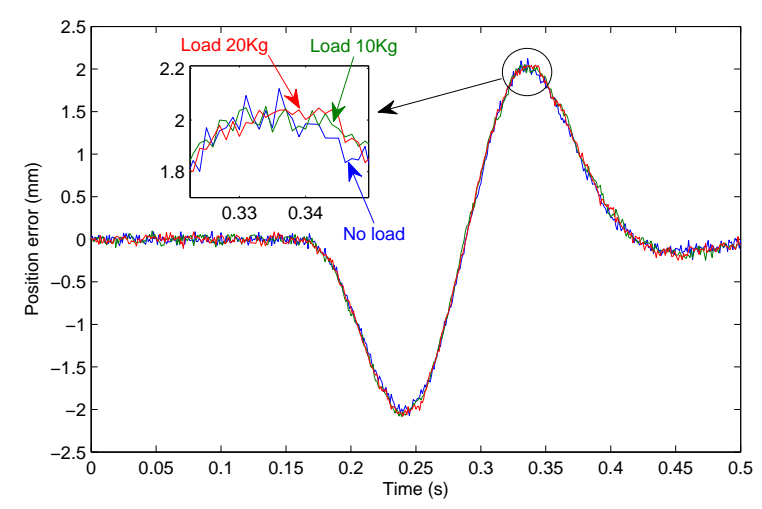

(b) Position error

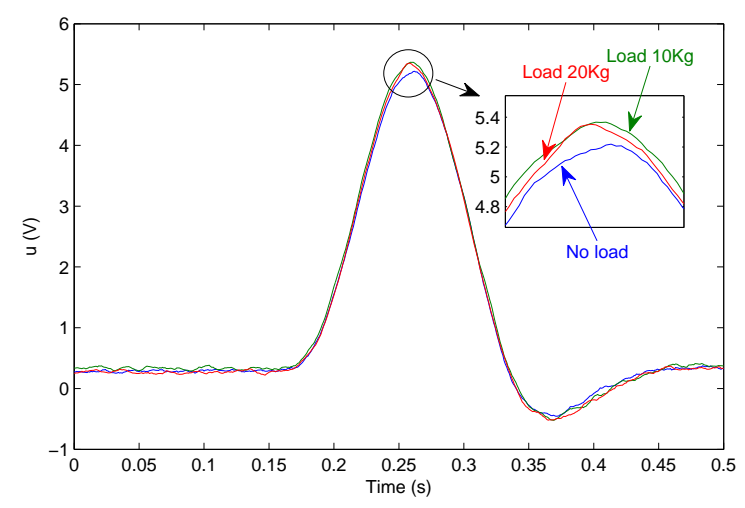

(c) Command $u$

Figure 5: Robustness experiment results obtained by the model-free control

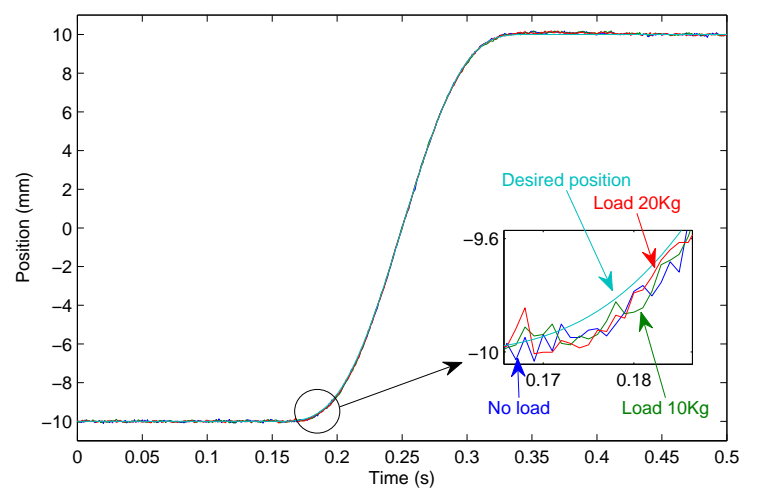

(a) Position tracking

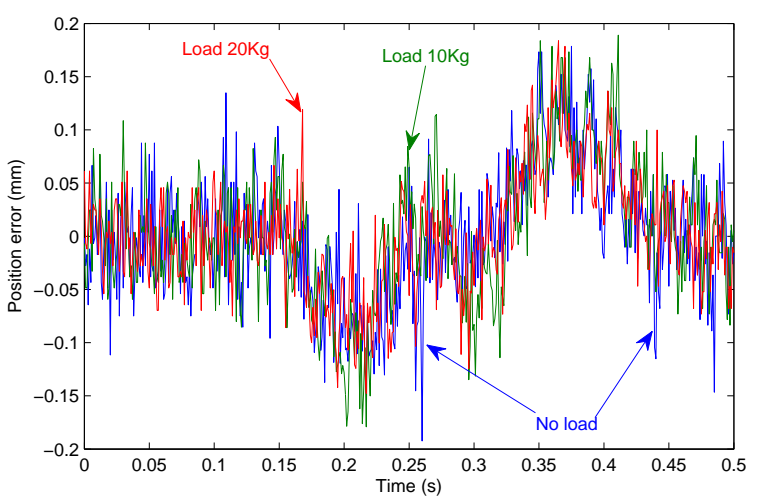

(b) Position error

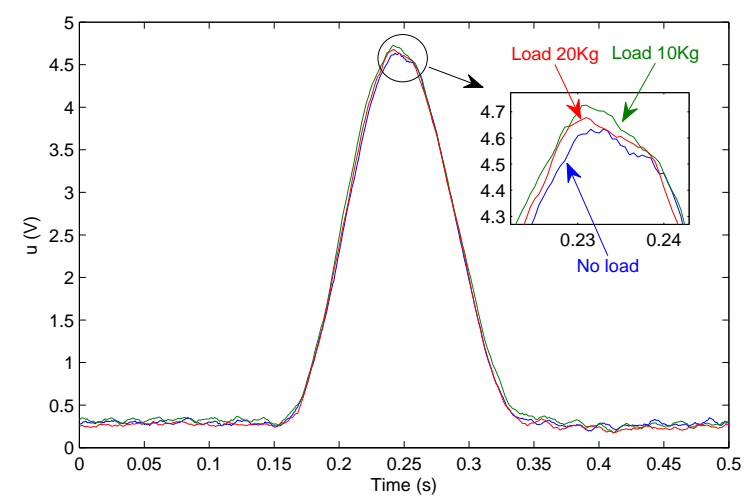

(c) Command $u$

Figure 6: Robustness experiment results obtained by the control with restricted model 
model when the load is changed. According to table 2, in all cases, the control with restricted model shows a higher precision in transient phase. In all cases, the average static error obtained from the two model-free based methods can be neglected. In conclusion, according to the experimental results, the controls based on the model-free method show very good robustness performances against the mass perturbation of the moving part.

\subsection{Experiments with different precisions of the esti- mated velocity}

The calculation of the uncertain ultra-local parameter $F$ (3) and (13) uses the estimated value of velocity obtained from the DAO2 algorithm. Hence, the control performances are associated with the estimation precision of the numeric differentiator which is influenced by the tuning gains of the DAO2. The phase shift of the estimated velocity becomes significant when the DAO2 gains are decreased, this leads actually to the precision degradation. However, the noise is obviously reduced. Unfortunately, as no direct measurements of velocity are available on the test rig, we are limited here to a qualitative analysis of the estimation precision and it is difficult to conclude on the estimation accuracy.

Figures 7, 8, and table 3 show the experimental results obtained with different precisions of the tuning gains of the $\mathrm{DAO} 2$ which is used here for velocity estimation. When these gains decrease, the estimated velocity will be less precise but will be less noisy. The other experiment parameters remain the same as those in section 3.1.

Table 3: Comparison summary of the experimental position error $(\mu \mathrm{m})$ with different precisions of DAO2: model-free control (MFC), control with restricted model (CRM), and proportional control (Prop.)

\begin{tabular}{|c|c|c|c|c|}
\hline \multirow{2}{*}{$\begin{array}{l}\text { Control } \\
\text { strategy }\end{array}$} & \multicolumn{2}{|c|}{ Transient phase } & \multicolumn{2}{|c|}{ Steady state } \\
\hline & Max. & $\mathrm{STD}^{c}$ & Mean & $\mathrm{STD}^{c}$ \\
\hline $\operatorname{MFC}\left(\mathrm{HG}-\mathrm{DAO} 2^{a}\right)$ & 2122 & 1344 & 0.06 & 39 \\
\hline $\mathrm{CRM}\left(\mathrm{HG}-\mathrm{DAO} 2^{a}\right)$ & 192 & 71 & 0.2 & 41 \\
\hline $\operatorname{MFC}\left(\mathrm{LG}-\mathrm{DAO} 2^{b}\right)$ & 1301 & 582 & 0.06 & 38 \\
\hline $\mathrm{CRM}\left(\mathrm{LG}-\mathrm{DAO} 2^{b}\right)$ & 1429 & 627 & -0.3 & 44 \\
\hline
\end{tabular}

For the model-free control (figure 8(a)), it can be noticed that the system needs more time to reach the high level position due to the degradation of the velocity precision. Some oscillations at low frequencies occur at the steady state. By comparing figures 7(d) and 8(d), it can be observed that the estimated velocities have less noise as the gains of the DAO2 decrease, but the phase shift becomes significant, which leads to fluctuations in the command calculations (figures 7(c) and 8(c)). Decreasing the estimated velocity precision leads to a more significant degradation on the tracking performance for the control with restricted model because this control law uses an approximate system model involving a term function

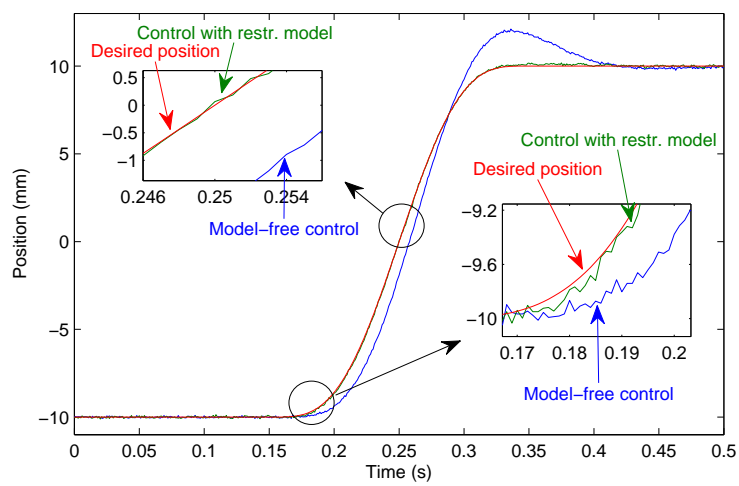

(a) Position tracking

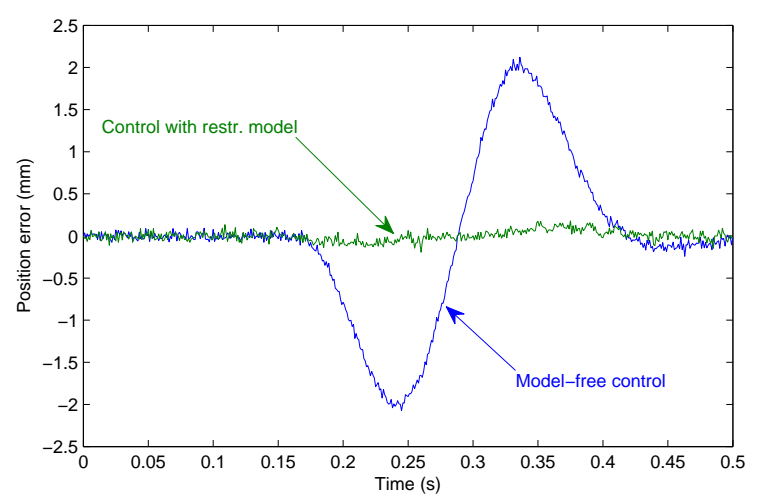

(b) Position error

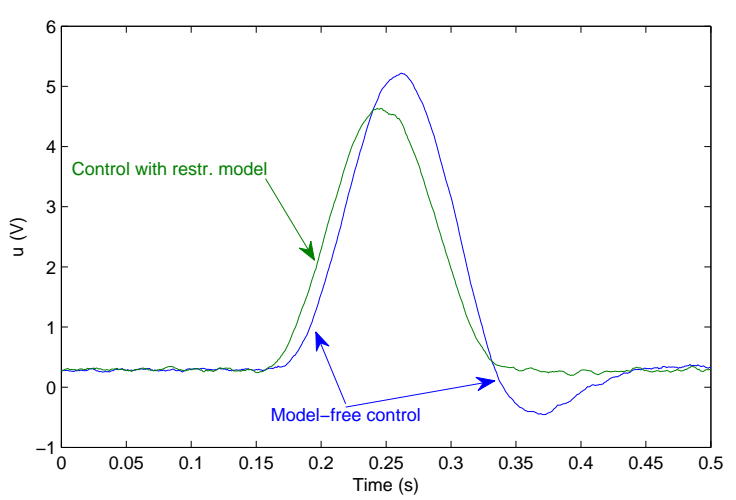

(c) Command $u$

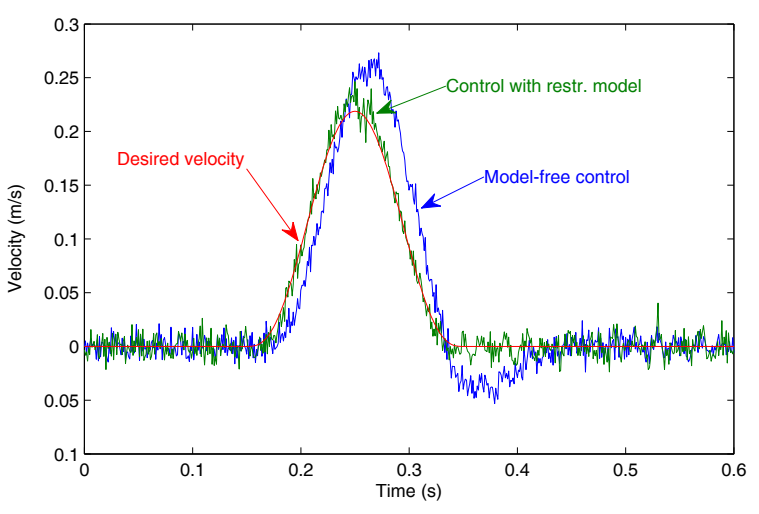

(d) Velocity evolution

Figure 7: Experimental results of the methods based on model-free control with high gain values of DAO2 


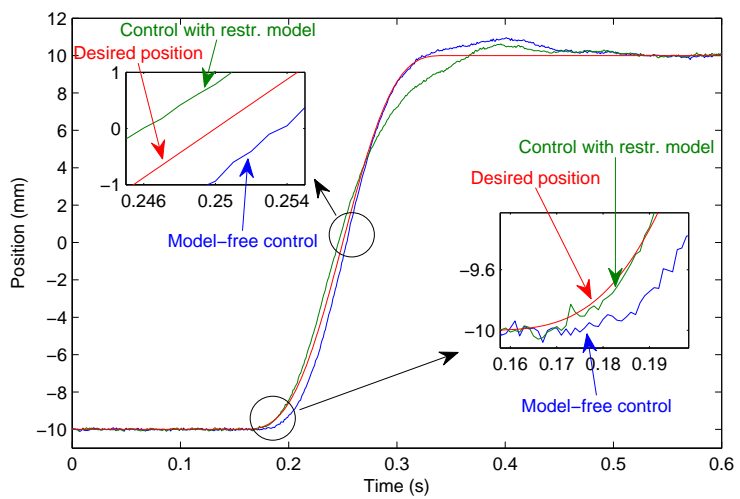

(a) Position tracking

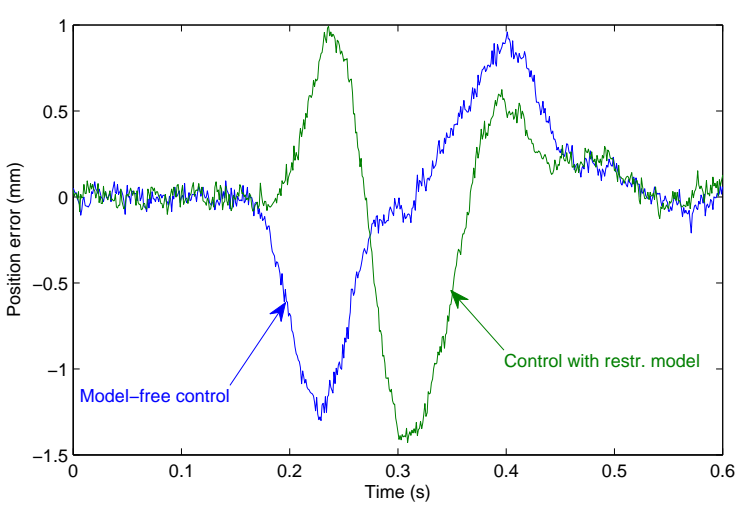

(b) Position error

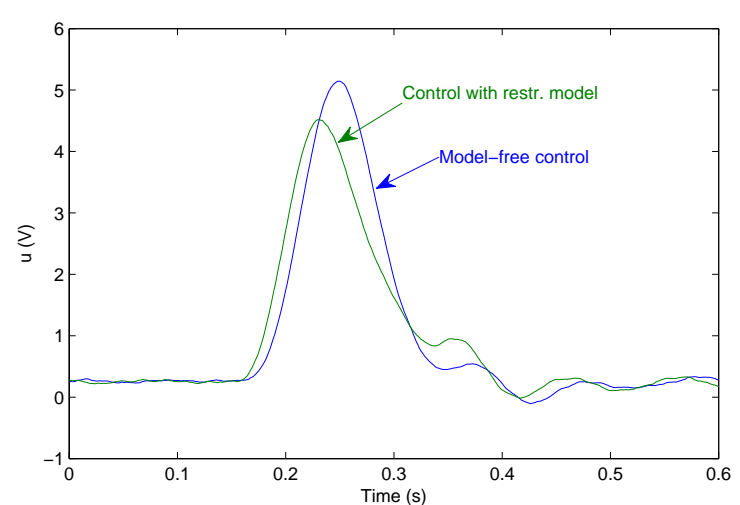

(c) Command $u$

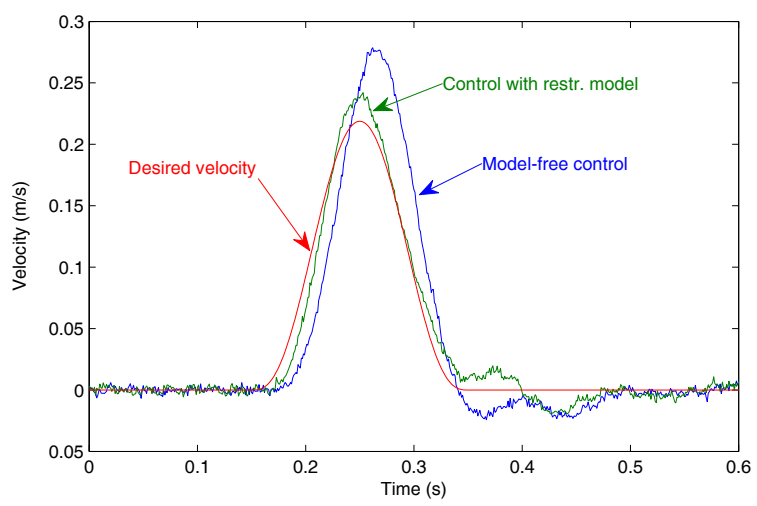

(d) Velocity evolution

Figure 8: Experimental results of the methods based on model-free control with low gain values of DAO2 of the velocity. On the other hand, although the tracking error of the model-free control decreases for low gain values of the $\mathrm{DAO} 2$, the control performance is actually degraded according to the fluctuations in the command (figure 8(c)) which implies a degradation of the system stability. In conclusion, for the two proposed control strategies, the tracking performances are degraded as the velocity becomes inaccurate. The precision of velocity estimation is then a critical factor to improve the performances of this kind of control strategies.

\section{Conclusion}

In this paper, the robustness performance of controls based on the model-free method has been studied throughout some experiments. These experiments results show that compared to the proportional control, the proposed control strategies have a good robustness performance against the mass change of the moving part. However, with the model-free based controls, it should be noticed that in order to get accurate tracking, the velocity estimation accuracy is an essential factor. To conclude, the model-free based control methods are easy to implement as they do not require a precise model of the system and have good robustness performances with high position tracking precision. This shows its high potential for application in industry.

\section{Nomenclature}

\begin{tabular}{lll}
\hline Designation & Denotation & Unit \\
\hline$y$ & Piston position & {$[\mathrm{m}]$} \\
$v$ & Actuator velocity & {$[\mathrm{m} / \mathrm{s}]$} \\
$a$ & Actuator acceleration & {$\left[\mathrm{m} / \mathrm{s}^{2}\right]$} \\
$u_{1,2}$ & Control input 1 or 2 & {$[\mathrm{~V}]$} \\
$P_{1,2}$ & Pressure in chamber 1 or 2 & {$[\mathrm{~Pa}]$} \\
$P_{T}$ & Tank pressure & {$[\mathrm{Pa}]$} \\
$P_{P}$ & Supply pressure & {$[\mathrm{Pa}]$} \\
$\Delta P_{n}$ & Rated valve pressure drop & {$[\mathrm{Pa}]$} \\
$u_{n}$ & Rated control input & {$[\mathrm{V}]$} \\
$Q_{n}$ & Rated flow & {$\left[\mathrm{m}{ }^{3} / \mathrm{s}\right]$} \\
$A$ & Piston active area & {$\left[\mathrm{m}{ }^{2}\right]$} \\
$b$ & Viscous coefficient & {$[\mathrm{N} /(\mathrm{m} / \mathrm{s})]$} \\
$\beta$ & Bulk modulus & {$[\mathrm{Pa}]$} \\
$V_{1,2}$ & Volume in chamber 1 or 2 & {$\left[\mathrm{~m}{ }^{3}\right]$} \\
$F_{f s}$ & Dry friction & {$[\mathrm{N}]$} \\
$F_{s d y n}$ & Dynamic dry friction & {$[\mathrm{N}]$} \\
$F_{C}$ & Coulomb friction & {$[\mathrm{N}]$} \\
$C$ & Friction index & {$[-]$} \\
\hline
\end{tabular}

\section{References}

[1] M. Fliess and C. Join. Commande sans modèle et commande à modèle restreint. $e-S T A, 5(4): 1-23,2008$.

[2] M. Fliess and C. Join. Model-free control and intelligent pid controllers: Towards a possible trivialization of nonlinear control. In Proceeding of 15th IFAC Symp. System Identif., Saint-Malo, 2009.

[3] M. Fliess, C. Join, and S. Riachy. Nothing is as practical as a good theory: Model-free control. In JN-JD-MACS, Marseille, 2011. 
[4] H.E. Merritt. Hydraulic Control Systems. John Wileys and Sons, Cincinnati, Ohio, 1967. ISBN 0-471-596175.

[5] D.H. Kim and T.-C. Tsao. A linearized electrohydraulic servovalve model for valve dynamics sensitivity analysis and control system design. Journal of Dynamic Systems, Measurement, and Control, 122(4):179-187, 2000.

[6] M. Smaoui, X. Brun, and D. Thomasset. A study on tracking position control of electropneumatic system using backstepping design. Control Engineering Practice, 14(8):923-933, Aug. 2006.

[7] Y. Xu, L. Sidhom, E. Bideaux, and M. Smaoui. Multivariable backstepping controls of a high bandwidth electro-hydraulic actuator. In Proceeding of Bath/ASME Symposium on Fluid Power \& Motion Control, Bath, UK, pages 397-410, Sep. 2012.

[8] A. Levant. Universal siso sliding-mode controllers with finite-time convergence. In IEEE Transaction on Automatic Control, Barcelona, volume 46, pages 14471451, 2001.

[9] G. Bartolini, A. Pisano, and E. Usai. A survey applications of second-order sliding mode control to mechanical systems. International Journal of Control, 76(9):875-892, June 2003.

[10] Y. Xu, E. Bideaux, S. Sesmat, and J.P. Simon. Dynamic effect of the intermediate block in a hydraulic control system. Journal of Mechanical Engineering and Automation, 2(6), Nov. 2012.

[11] Y. Xu, E. Bideaux, and D. Thomasset. Model-free control and control with restricted model of a high performance hydraulic system. In 8th International Conference on Fluid Power Transmission and Control, Hangzhou, China, 2013.

[12] L. Sidhom, M. Smaoui, D. Thomasset, X. Brun, and E. Bideaux. Adaptive higher order sliding modes for two-dimensional derivative estimation. In Proceeding of 18th World Congress of the International Federation of Automatic Control (IFAC), Milano, 2011.

[13] MOOG. Servovalves with integrated electronics d765 series. Technical report, MOOG Inc. 